\title{
Synthesis of New Chiral Benzimidazolylidene-Rh Complexes and Their Application in Asymmetric Addition Reactions of Organoboronic Acids to Aldehydes
}

\author{
Weiping $\mathrm{He}^{1,3}$, Bihui Zhou ${ }^{1,3}$, Jie $\mathrm{Li}^{1, *}$ and Jianyou $\mathrm{Shi}^{2, *}$ \\ 1 School of Medicine, Zhejiang University City College, No. 48, Huzhou Road, Hangzhou 310015, China; \\ wphe2016@163.com (W.H.); 18868816170@163.com (B.Z.) \\ 2 Individualized Medication Key Laboratory of Sichuan Province, Sichuan Academy of Medical Science \& \\ Sichuan Provincial People's Hospital, School of Medicine, Center for Information in Medicine, \\ University of Electronic Science and Technology of China, Chengdu 610072, China \\ 3 College of Pharmaceutical Sciences, Zhejiang University, Hangzhou 310058, China \\ * Correspondence: lijie@zucc.edu.cn (J.L.); shijianyoude@126.com (J.S.); \\ Tel.: +86-571-8801-6565 (J.L.); +86-177-0813-0657 (J.S.)
}

Academic Editor: Georgiy B. Shul'pin

Received: 29 July 2016; Accepted: 25 August 2016; Published: 3 September 2016

\begin{abstract}
A series of novel chiral N-heterocyclic carbene rhodium complexes (NHC-Rh) based on benzimidazole have been prepared, and all of the NHC-Rh complexes were fully characterized by NMR and mass spectrometry. These complexes could be used as catalysts for the asymmetric 1,2-addition of organoboronic acids to aldehydes, affording chiral diarylmethanols with high yields and moderate enantioselectivities.
\end{abstract}

Keywords: $N$-heterocycliccarbene; benzimidazolium; Rh-catalyzed; asymmetric 1,2-addition

\section{Introduction}

Since $N$-heterocycliccarbenes (NHCs) are excellent $\sigma$-donors, and their metal complexes show higher air and thermal stability than phosphane ligands. NHCs are now well established as efficient alternatives to phosphane ligands [1-10]. Much work has been devoted to the design and development of carbene compounds with new structures to tune their steric and electronic properties, and also to their application in organometallic catalysis. As excellent ligands for transition metals, NHCs have found multiple applications in some of the most important catalytic transformations in the chemical industry. As a logical extension of this development, chiral NHC ligands and their application in asymmetric catalysis are receiving increasing attention [11-16]. Despite considerable efforts devoted to this field, the design and synthesis of novel chiral NHCs to enhance their enantioselectivity is still a challenge.

Enantioenriched diarylmethanols are the structural core unit in a considerable number of bioactive compounds and pharmaceuticals [17-20]. The Rh-catalyzed enantioselective arylation of aromatic aldehydes with organoboronic acids has emerged as a direct and economical route for the synthesis of enantiomerically-enriched diarylmethanols [21]. In 1998, Miyaura and co-workers initially reported the enantioselective Rh-catalyzed addition of phenylboronic acid to naphthaldehyde by using the (S)-MeO-MOP ligand, giving naphthyphenylmethanol in 78\% yield and 41\% ee [22]. Since then, considerable efforts have been made in this type of reaction [23-30]. However, examples of using chiral $N$-heterocycliccarbenes in the ligand-catalyzed asymmetric arylation of aldehydes are rare [31-35]. Therefore, developing new chiral $N$-heterocycliccarbene ligands for the asymmetric 1,2-addition of 
organoboronic acid to aldehydes is an important synthetic goal. The above-mentioned findings, and our interests in NHCs and C-C forming reactions triggered our efforts to develop new NHC ligands for application in homogeneous catalysis. After our recent report of the synthesis of several chiral benzimidazolium salts for the in situ Rh-catalyzed asymmetric arylation of aldehydes [36], we herein report the synthesis of a series of new NHC-Rh complexes based on benzimidazole and their application in the asymmetric 1,2-addition of arylboronic acids to aldehydes.

\section{Results}

The synthetic route to the new NHC-Rh complexes based on the benzimidazole skeleton is shown in Schemes 1 and 2. The NHC complexes were synthesized from enantiomerically-pure benzimidazolium salts (1a-g), which in turn can be prepared by following our previous articles [36,37]. Among the NHC precursors prepared, compounds $1 \mathbf{c}$ and $\mathbf{1 d}$ were new and are reported for the first time in this paper. In the next step, the mild transmetalation developed by Wang and Lin was adopted to prepare rhodium(I) complexes of $\mathbf{1 a} \mathbf{a}$. According to this strategy, the benzimidazolium salts $\mathbf{1}$ were treated with $\mathrm{Ag}_{2} \mathrm{O}$ in anhydrous $\mathrm{CH}_{2} \mathrm{Cl}_{2}$ at room temperature in the darkness. Then direct addition of $\left[\mathrm{Rh}(\mathrm{COD}) \mathrm{Cl}_{2}\right.$ to the freshly prepared solution of silver complexes yielded the corresponding chiral complexes $2 \mathbf{a}-\mathbf{g}$ upon workup, which could be purified by chromatography on silica gel (Scheme 2). The complexes were characterized by ${ }^{1} \mathrm{H} N M R,{ }^{13} \mathrm{C} N M R$, and high-resolution mass spectrometry (HRMS), and the absence of an $\mathrm{N}-\mathrm{C}_{\mathrm{NHC}}-\mathrm{N}$ resonance in the ${ }^{1} \mathrm{H}$ NMR spectra confirmed the formation of the carbene complexes.
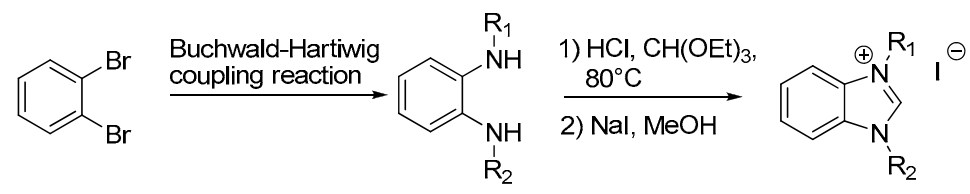

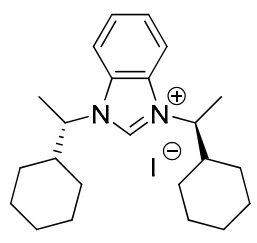

$1 \mathbf{a}$

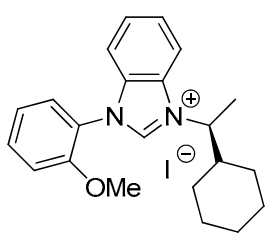

$1 e$

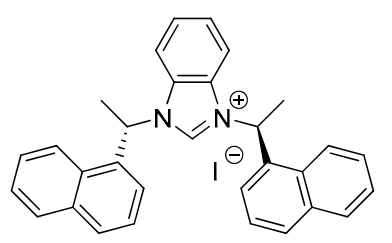

$1 \mathrm{~b}$

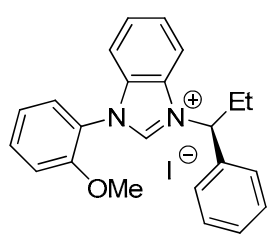

$1 f$

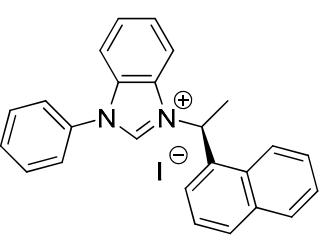

$1 \mathrm{c}$

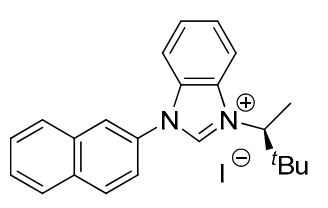

$1 g$

Scheme 1. Synthesis of benzimidazolium salts 1a-g.

With the chiral NHC-Rh complexes in hand, we examined their application in the asymmetric addition of organoboronic acids to aldehydes. Firstly, all of the NHC-Rh complexes were tested in enantioselective phenylation of 2-naphthaldehyde (3a) with $\mathrm{PhB}(\mathrm{OH})_{2}$. The reaction was performed with $3.0 \mathrm{~mol} \%$ of NHC-Rh complex in DME/ $\mathrm{H}_{2} \mathrm{O}(5: 1)$ at $80{ }^{\circ} \mathrm{C}$ for $12 \mathrm{~h}$. As shown in Table 1, diarylmethanol $4 \mathbf{a}$ was obtained in high yield with each of the NHC-Rh complexes, and compound $\mathbf{2 g}$ gave the best result $(18 \%$ ee). 


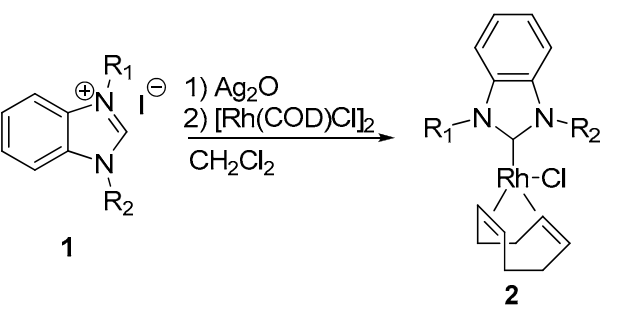

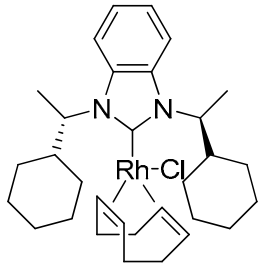

$2 a$

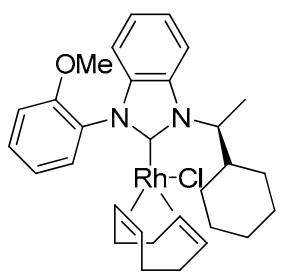

$2 e$

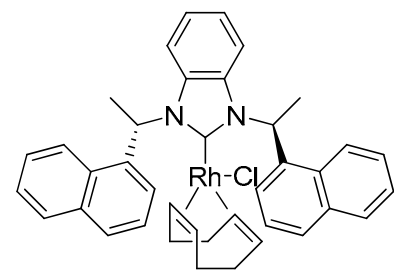

$2 b$

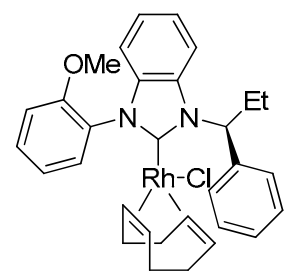

$2 f$

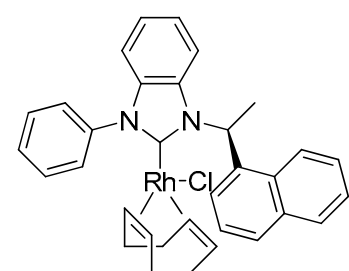

2c<smiles>CC(C)[C@H](C)N1c2ccccc2N(c2ccc3ccccc3c2)C1[R7](Cl)(C1=CCCC1)C1CCC1</smiles>

$2 g$

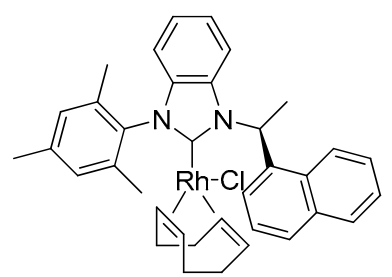

2d

Scheme 2. Synthesis of $\mathrm{N}$-heterocyclic carbene-rhodium NHC-Rh complexes $\mathbf{2 a - g}$.

Table 1. Comparison of NHC-Rh complexes.

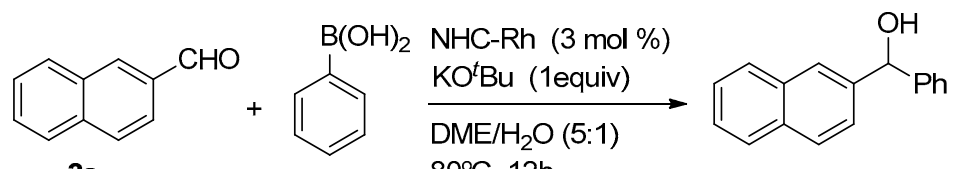

$3 a$

$80^{\circ} \mathrm{C}, 12 \mathrm{~h}$

$4 a$

\begin{tabular}{cccc}
\hline Entry $^{\mathbf{a}}$ & Ligand & Yield (\%) $^{\mathbf{b}}$ & ee (\%) \\
\hline 1 & $2 \mathrm{c}$ & 99 & 3 \\
2 & $2 \mathrm{~b}$ & 99 & 1 \\
3 & $2 \mathrm{c}$ & 99 & 6 \\
4 & $2 \mathrm{~d}$ & 98 & 17 \\
5 & $2 \mathrm{e}$ & 99 & 3 \\
6 & $2 \mathrm{f}$ & 98 & 3 \\
7 & $2 \mathrm{~g}$ & 99 & 18 \\
8 & no catalyst & - & - \\
\hline
\end{tabular}

a Reaction condition: ligand ( $3 \mathrm{~mol} \%), \mathrm{KO}^{t} \mathrm{Bu}$ (1 equiv.), arylboronic acids (2 equiv.), $\mathrm{N}_{2}, \mathrm{DME} / \mathrm{H}_{2} \mathrm{O}(5: 1)$, $80^{\circ} \mathrm{C}, 12 \mathrm{~h}$; ${ }^{\mathrm{b}}$ Isolated yields; ${ }^{\mathrm{c}}$ Determined by chiral HPLC (CHIRALCEL OD Column) analysis.

We then optimized the experimental conditions using $\mathbf{2 g}$ as catalyst. By screening bases in $\mathrm{DME} / \mathrm{H}_{2} \mathrm{O}$ (5:1), we found that the addition of excess KF (6.0 equiv.) significantly improved the enantioselectivity as well as yield (Table 2, entry 6). Next, variation of the solvent indicated that the 5:1 mixture of EtOH/DME was the best choice of solvent (Table 2, entry 16). Further screening of reaction temperature showed that lower temperature afforded the product with similar enantioselectivities but inferior yields (Table 3, entries 20-22). 
Table 2. Optimization of the reaction conditions.

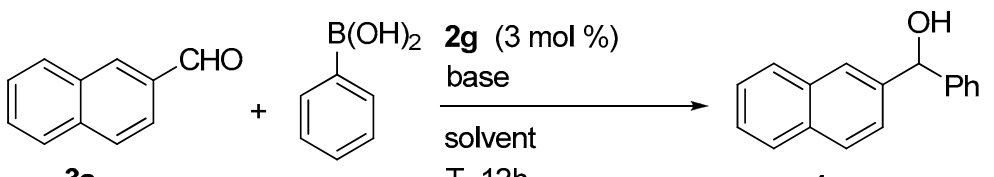

$\mathrm{T}, 12 \mathrm{~h}$

\begin{tabular}{|c|c|c|c|c|c|}
\hline Entry $^{a}$ & Base & Solvent & Temperature $\left({ }^{\circ} \mathrm{C}\right)$ & Yield $(\%)^{b}$ & ee $(\%)^{c}$ \\
\hline 1 & $\mathrm{NaO}^{t} \mathrm{Bu}$ & $\mathrm{DME} / \mathrm{H}_{2} \mathrm{O}(5: 1)$ & 80 & 99 & 18 \\
\hline 2 & $\mathrm{LiO}^{t} \mathrm{Bu}$ & $\mathrm{DME} / \mathrm{H}_{2} \mathrm{O}(5: 1)$ & 80 & 42 & 29 \\
\hline 3 & $\mathrm{LiOH}$ & $\mathrm{DME} / \mathrm{H}_{2} \mathrm{O}(5: 1)$ & 80 & 99 & 17 \\
\hline 4 & KF (1 equiv.) & $\mathrm{DME} / \mathrm{H}_{2} \mathrm{O}(5: 1)$ & 80 & 70 & 32 \\
\hline 5 & KF (3 equiv.) & $\mathrm{DME} / \mathrm{H}_{2} \mathrm{O}(3: 1)$ & 80 & 80 & 32 \\
\hline 6 & KF (6 equiv.) & $\mathrm{DME} / \mathrm{H}_{2} \mathrm{O}(5: 1)$ & 80 & 99 & 32 \\
\hline 7 & KF (6 equiv.) & $\mathrm{DME} / \mathrm{H}_{2} \mathrm{O}(10: 1)$ & 80 & 99 & 14 \\
\hline 8 & KF (6 equiv.) & $\mathrm{DME} / \mathrm{H}_{2} \mathrm{O}(3: 1)$ & 80 & 99 & 18 \\
\hline 9 & KF (6 equiv.) & Toluene $/ \mathrm{H}_{2} \mathrm{O}(5: 1)$ & 80 & 99 & 22 \\
\hline 10 & KF (6 equiv.) & $\mathrm{MeOH} / \mathrm{DME}(5: 1)$ & 80 & 99 & 25 \\
\hline 11 & KF (6 equiv.) & $t-\mathrm{BuOH} / \mathrm{MeOH}(5: 1)$ & 80 & 99 & 24 \\
\hline 12 & KF (6 equiv.) & $\mathrm{MeOH}$ & 80 & 99 & 21 \\
\hline 13 & KF (6 equiv.) & $i$-PrOH & 80 & 99 & 34 \\
\hline 14 & KF (6 equiv.) & $t-\mathrm{BuOH} / \mathrm{EtOH}(5: 1)$ & 80 & 99 & 25 \\
\hline 15 & KF (6 equiv.) & DME & 80 & 93 & 9 \\
\hline 16 & KF (6 equiv.) & EtOH/DME (5:1) & 80 & 99 & 35 \\
\hline 17 & KF (6 equiv.) & $\mathrm{EtOH}$ & 80 & 99 & 32 \\
\hline 18 & KF (6 equiv.) & Dioxane & 80 & 94 & 17 \\
\hline 19 & KF (6 equiv.) & $i$-PrOH/DME (5:1) & 80 & 99 & 34 \\
\hline 20 & KF (6 equiv.) & EtOH/DME (5:1) & 50 & - & - \\
\hline 21 & KF (6 equiv.) & $i-\mathrm{PrOH}$ & 50 & 43 & 33 \\
\hline 22 & KF (6 equiv.) & $i-\mathrm{PrOH} / \mathrm{DME}(5: 1)$ & 50 & 47 & 36 \\
\hline
\end{tabular}

${ }^{a}$ Reaction condition: $2 \mathrm{~g}$ ( $3 \mathrm{~mol} \%$ ), base (1 equiv.), arylboronic acids ( 2 equiv.), $\mathrm{N}_{2}, 80^{\circ} \mathrm{C}, 12 \mathrm{~h}$; ${ }^{\mathrm{b}}$ Isolated yields;

${ }^{c}$ Determined by chiral HPLC (CHIRALCEL OD Column) analysis.

Table 3. Scope of methodology.

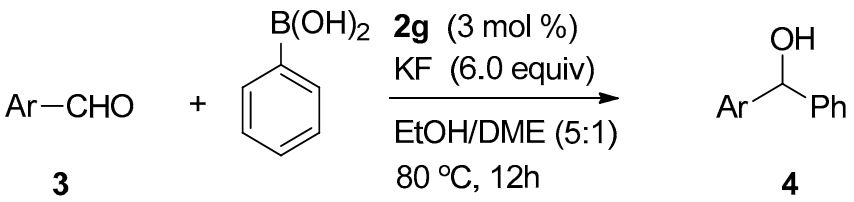

\begin{tabular}{cccc}
\hline Entry $^{\mathbf{a}}$ & Ar $_{\mathbf{1}}$ & Yield (\%) $^{\mathbf{b}}$ & ee (\%) \\
\hline 1 & 1-Naphthyl 3b & $97 \mathbf{4 b}$ & 43 \\
2 & 2-MeOPh 3c & $99 \mathbf{b}$ & 37 \\
3 & 4-MeOPh 3d & $85 \mathbf{4 d}$ & 46 \\
4 & 4-CF 3 Ph 3e & $94 \mathbf{4 e}$ & 40 \\
5 & 3,4-DiMePh 3f & $99 \mathbf{4 f}$ & 28 \\
6 & 4-EtPh 3g & $99 \mathbf{4} \mathbf{g}$ & 36 \\
7 & 2-FPh 3h & $93 \mathbf{4 h}$ & 38 \\
8 & 3,5-DiFPh 3i & $88 \mathbf{4 i}$ & 28 \\
9 & 4-NO $2 \mathrm{Ph} 3 \mathbf{j}$ & $94 \mathbf{4 j}$ & 28 \\
10 & 2-thienyl 3k & $99 \mathbf{j k}$ & 18 \\
11 & 2-furyl 31 & $98 \mathbf{4 l}$ & 19
\end{tabular}

a Reaction condition: $2 \mathrm{~g}$ ( $3 \mathrm{~mol} \%), \mathrm{KF}$ (6.0 equiv.), arylboronic acids (2 equiv.), EtOH/DME (5:1), $\mathrm{N}_{2}, 80^{\circ} \mathrm{C}$, $12 \mathrm{~h} ;{ }^{\mathrm{b}}$ Isolated yields; ${ }^{\mathrm{c}}$ Determined by chiral HPLC (CHIRALCEL OD or AD Column) analysis.

Having optimized reaction conditions, we examined the reactions with various aldehydes, and the results are summarized in Table 3. The arylations with either electron-rich or electron-deficient benzaldehydes proceeded smoothly to afford the corresponding diarylmethanols in excellent yields and moderate enantioselectivities. The best enantioselectivity was obtained starting from $o$-anisaldehyde ( $46 \%$ ee, entry 3 ). 


\section{Materials and Methods}

\subsection{General}

MS spectra were measured on a Finnigan LCQDECA XP instrument (ThermoFinnigan Co., California, CA, USA) and an Agilent Q-TOF 1290LC/6224 MS system (Aglient Technologies Inc., California, CA, USA); ${ }^{1} \mathrm{H}$ and ${ }^{13} \mathrm{C}$ NMR spectra were obtained on Bruker AVANCE III $500 \mathrm{MHz}$ and $600 \mathrm{MHz}$ spectrometers (Bruker Co., Faellanden, Switzerland) with TMS as the internal standard; silica gel $\mathrm{GF}_{254}$ and $\mathrm{H}$ (10-40 mm, Qingdao Marine Chemical Factory, Qingdao, China) were used for TLC and CC. Unless otherwise noted, all reactions were carried out under an atmosphere of argon or nitrogen.

\subsection{Preparation of Benzimidazolium Salt $\mathbf{1 a}-\mathbf{g}$}

The NHC precursors 1a-g were synthesized following our previous paper [36], and the ${ }^{1} \mathrm{H}$ NMR spectra of $\mathbf{1 a}-\mathbf{b}$ and $\mathbf{1 e}-\mathbf{f}$ were identical to those reported in the literature [36,37]. 1c: ${ }^{1} \mathrm{H}$ NMR $\left(500 \mathrm{MHz}, \mathrm{CDCl}_{3}\right) \delta: 11.18(\mathrm{~s}, 1 \mathrm{H}), 8.24-7.08(\mathrm{~m}, 14 \mathrm{H}), 2.53(\mathrm{~d}, J=6.9 \mathrm{~Hz}, 3 \mathrm{H}), 2.39(\mathrm{~s}, 3 \mathrm{H}), 2.07(\mathrm{~s}, 3 \mathrm{H})$, $2.00(\mathrm{~s}, 3 \mathrm{H}) .1 \mathrm{~d}:{ }^{1} \mathrm{H}$ NMR $\left(500 \mathrm{MHz}, \mathrm{CDCl}_{3}\right) \delta: 10.73(\mathrm{~s}, 1 \mathrm{H}), 8.21-7.42(\mathrm{~m}, 16 \mathrm{H}), 7.11(\mathrm{q}, J=6.8 \mathrm{~Hz}, 1 \mathrm{H})$, $2.56(\mathrm{~d}, J=6.8 \mathrm{~Hz}, 3 \mathrm{H})$.

\subsection{Preparation of NHC-Rh Complexes $\mathbf{2 a -} \mathbf{g}$}

To a solution of imidazolinium salt $1 \mathrm{a}(364.0 \mathrm{mg}, 1.00 \mathrm{mmol})$ in $\mathrm{CH}_{2} \mathrm{Cl}_{2}(25 \mathrm{~mL})$ was added silver(I) oxide ( $115.9 \mathrm{mg}, 0.50 \mathrm{mmol}$ ) in one portion. The suspension was stirred for $3 \mathrm{~h}$ in the darkness, during which the black color gradually diminished. The reaction mixture was filtered through a small pad of Celite, $\left[\mathrm{Rh}(\mathrm{COD}) \mathrm{Cl}_{2}(246.5 \mathrm{mg}, 0.50 \mathrm{mmol})\right.$ was added in one portion, and the reaction mixture was stirred for an additional $16 \mathrm{~h}$. The solvent was evaporated, and the residue was purified by flash chromatography on silica gel with $\mathrm{CH}_{2} \mathrm{Cl}_{2}$ as eluent. After evaporation of volatiles, the residue was purified by column chromatography $\left(\mathrm{CH}_{2} \mathrm{Cl}_{2}\right)$ to give $2 \mathrm{a}\left(515.0 \mathrm{mg}, 88 \%\right.$ yield). ${ }^{1} \mathrm{H} \mathrm{NMR}(500 \mathrm{MHz}$, $\left.\mathrm{CDCl}_{3}\right) \delta: 7.52-7.43(\mathrm{~m}, 2 \mathrm{H}), 7.19-7.08(\mathrm{~m}, 2 \mathrm{H}), 5.86(\mathrm{~m}, 1 \mathrm{H}), 5.73(\mathrm{~m}, 1 \mathrm{H}), 5.18(\mathrm{~m}, 1 \mathrm{H}), 5.04(\mathrm{~m}, 1 \mathrm{H})$, $3.66(\mathrm{t}, J=7.2 \mathrm{~Hz}, 1 \mathrm{H}), 3.41(\mathrm{~d}, J=7.5 \mathrm{~Hz}, 1 \mathrm{H}), 2.58-1.79(\mathrm{~m}, 15 \mathrm{H}), 1.71(\mathrm{~m}, 6 \mathrm{H}), 1.52-0.86(\mathrm{~m}, 15 \mathrm{H})$; ${ }^{13} \mathrm{C}$ NMR $\left(125 \mathrm{MHz}, \mathrm{CDCl}_{3}\right) \delta: 134.17,133.92,121.77,121.53,112.10,112.01,98.81,98.76,98.66,98.61$, $77.28,76.78,68.79,68.67,67.40,67.28,63.73,62.82,42.57,42.35,33.29,32.35,32.07,31.93,31.34,30.68$, $30.37,29.70,29.36,28.23,26.53,26.42,26.40,26.37,26.05,25.94,22.70,19.06,18.43,14.12$; HR-ESIMS: $m / z 549.2792$ [M-Cl] $]^{+}$(calcd. for $\mathrm{C}_{31} \mathrm{H}_{46} \mathrm{~N}_{2} \mathrm{Rh}, 549.2716$ ).

Analogous compounds $\mathbf{2} \mathbf{b}-\mathbf{g}$ were prepared according to the similar procedure for $\mathbf{2} \mathbf{a}$. HR-ESIMS, ${ }^{1} \mathrm{H}$ and ${ }^{13} \mathrm{C}$ NMR data see Supplementary Materials. 2b: $97 \%$ yield; ${ }^{1} \mathrm{H}$ NMR $\left(500 \mathrm{MHz}, \mathrm{CDCl}_{3}\right)$ $\delta: 9.08(\mathrm{q}, J=7.3 \mathrm{~Hz}, 1 \mathrm{H}), 8.57(\mathrm{~m}, 2 \mathrm{H}), 8.03-7.51(\mathrm{~m}, 9 \mathrm{H}), 7.36(\mathrm{~m}, 5 \mathrm{H}), 7.18(\mathrm{~m}, 2 \mathrm{H}), 7.11-7.00$ $(\mathrm{m}, 1 \mathrm{H}), 5.16(\mathrm{~m}, 1 \mathrm{H}), 5.03(\mathrm{~m}, 1 \mathrm{H}), 3.38(\mathrm{~s}, 1 \mathrm{H}), 3.02(\mathrm{t}, J=7.2 \mathrm{~Hz}, 1 \mathrm{H}), 2.67(\mathrm{~d}, J=7.2 \mathrm{~Hz}, 3 \mathrm{H})$, $2.35(\mathrm{~m}, 1 \mathrm{H}), 2.25(\mathrm{~d}, J=7.3 \mathrm{~Hz}, 3 \mathrm{H}), 2.15-1.98(\mathrm{~m}, 1 \mathrm{H}), 1.96-1.77(\mathrm{~m}, 2 \mathrm{H}), 1.55(\mathrm{~m}, 1 \mathrm{H}), 1.51-1.41$ $(\mathrm{m}, 1 \mathrm{H}), 1.20(\mathrm{~d}, J=10.3 \mathrm{~Hz}, 1 \mathrm{H}), 0.95-0.88(\mathrm{~m}, 1 \mathrm{H}) ;{ }^{13} \mathrm{C} \mathrm{NMR}\left(125 \mathrm{MHz}, \mathrm{CDCl}_{3}\right) \delta: 138.83,136.20$, 135.84, 134.96, 134.13, 134.06, 130.80, 130.03, 129.10, 129.03, 128.77, 128.01, 127.07, 126.59, 126.12, 126.03, $125.21,125.14,124.19,123.90,123.73,122.35,122.32,122.24,113.93,112.26,99.20,97.82,77.28,76.78$, 70.74, 70.62, 67.45, 67.33, 61.11, 56.76, 32.68, 31.94, 31.75, 29.71, 29.35, 27.39, 22.70, 22.33, 20.83, 14.13; HR-ESIMS: $m / z$ 529.1152 [M-Cl-COD] ${ }^{+}$(calcd. for $\mathrm{C}_{31} \mathrm{H}_{26} \mathrm{~N}_{2} \mathrm{Rh}, 529.1151$ ). $2 \mathrm{c}$ : 91\% yield; ${ }^{1} \mathrm{H}$ NMR $\left(500 \mathrm{MHz}, \mathrm{CDCl}_{3}\right) \delta: 8.76-8.56(\mathrm{~m}, 2 \mathrm{H}), 8.21(\mathrm{~d}, J=5.5 \mathrm{~Hz}, 2 \mathrm{H}), 7.96(\mathrm{~d}, J=8.1 \mathrm{~Hz}, 1 \mathrm{H}), 7.78(\mathrm{~m}, 3 \mathrm{H}), 7.61$ $(\mathrm{m}, 4 \mathrm{H}), 7.44(\mathrm{~d}, J=8.0 \mathrm{~Hz}, 1 \mathrm{H}), 7.39-7.24(\mathrm{~m}, 4 \mathrm{H}), 5.01(\mathrm{~s}, 2 \mathrm{H}), 2.60-2.52(\mathrm{~m}, 1 \mathrm{H}), 2.48(\mathrm{~m}, 1 \mathrm{H}), 2.31(\mathrm{~d}$, $J=7.4 \mathrm{~Hz}, 3 \mathrm{H}), 1.91-1.80(\mathrm{~m}, 1 \mathrm{H}), 1.70-1.61(\mathrm{~m}, 1 \mathrm{H}), 1.55-1.40(\mathrm{~m}, 2 \mathrm{H}), 1.31(\mathrm{~m}, 2 \mathrm{H}), 1.09-0.99(\mathrm{~m}$, $1 \mathrm{H}), 0.65(\mathrm{~m}, 1 \mathrm{H}) ;{ }^{13} \mathrm{C} \mathrm{NMR}\left(125 \mathrm{MHz}, \mathrm{CDCl}_{3}\right) \delta: 139.48,138.06,136.15,134.82,133.99,130.09,129.10$, $128.97,128.52,127.98,127.29,126.78,126.14,125.25,123.87,123.01,122.74,122.15,113.24,111.01,99.67$, $99.62,98.81,98.76,77.28,76.77,69.64,69.53,68.89,68.77,59.83,53.42,31.98,31.77,29.70,28.07,27.96$, 20.95; HR-ESIMS: $m / z 559.2367$ [M-Cl] ${ }^{+}$(calcd. for $\mathrm{C}_{33} \mathrm{H}_{32} \mathrm{~N}_{2} \mathrm{Rh}, 559.1621$ ). 2d: 89\% yield; ${ }^{1} \mathrm{H}$ NMR $\left(500 \mathrm{MHz}, \mathrm{CDCl}_{3}\right) \delta: 8.91(\mathrm{q}, J=7.3 \mathrm{~Hz}, 1 \mathrm{H}), 8.65(\mathrm{~d}, J=8.6 \mathrm{~Hz}, 1 \mathrm{H}), 7.96(\mathrm{~d}, J=8.1 \mathrm{~Hz}, 1 \mathrm{H}), 7.85-7.69$ 
$(\mathrm{m}, 3 \mathrm{H}), 7.61(\mathrm{t}, J=7.5 \mathrm{~Hz}, 1 \mathrm{H}), 7.33-7.27(\mathrm{~m}, 2 \mathrm{H}), 7.24-7.11(\mathrm{~m}, 3 \mathrm{H}), 7.01-6.86(\mathrm{~m}, 2 \mathrm{H}), 4.98(\mathrm{~m}, 1 \mathrm{H})$, 4.89-4.78 (m, 1H), 3.07-2.93 (m, 1H), $2.72(\mathrm{t}, J=7.2 \mathrm{~Hz}, 1 \mathrm{H}), 2.51(\mathrm{~s}, 3 \mathrm{H}), 2.43(\mathrm{~s}, 3 \mathrm{H}), 2.37(\mathrm{~d}, J=7.3 \mathrm{~Hz}$, $3 \mathrm{H}), 2.02-1.88(\mathrm{~m}, 1 \mathrm{H}), 1.65(\mathrm{~m}, 4 \mathrm{H}), 1.54-1.28(\mathrm{~m}, 4 \mathrm{H}), 1.10(\mathrm{~s}, 1 \mathrm{H}), 0.67(\mathrm{~m}, 1 \mathrm{H}) ;{ }^{13} \mathrm{C} \mathrm{NMR}(125 \mathrm{MHz}$, $\left.\mathrm{CDCl}_{3}\right) \delta: 140.28,139.09,138.04,136.73,135.17,134.61,134.05,132.92,130.17,130.04,128.93,128.37$, $127.78,126.65,126.11,125.33,124.15,122.82,122.70,121.60,113.30,110.80,98.82,98.77,98.48,98.42$, $77.29,76.78,69.57,69.45,67.98,67.86,59.99,32.79,31.94,31.32,29.70,29.67,29.37,28.96,27.19,22.70$, 21.18, 21.06, 19.68, 17.72, 14.12; HR-ESIMS: $m / z$ 601.4336 [M-Cl] ${ }^{+}$(calcd. for $\mathrm{C}_{36} \mathrm{H}_{38} \mathrm{~N}_{2} \mathrm{Rh}, 601.2090$ ). 2e: $83 \%$ yield; ${ }^{1} \mathrm{H}$ NMR $\left(500 \mathrm{MHz}, \mathrm{CDCl}_{3}\right) \delta: 8.45(\mathrm{~m}, 1 \mathrm{H}), 7.60-7.47(\mathrm{~m}, 2 \mathrm{H}), 7.29(\mathrm{~m}, 1 \mathrm{H}), 7.22-7.08$ $(\mathrm{m}, 3 \mathrm{H}), 6.95(\mathrm{~d}, J=7.9 \mathrm{~Hz}, 1 \mathrm{H}), 5.74-5.64(\mathrm{~m}, 1 \mathrm{H}), 5.03-4.88(\mathrm{~m}, 2 \mathrm{H}), 3.78(\mathrm{~d}, J=6.1 \mathrm{~Hz}, 3 \mathrm{H}), 3.56$ (s, 1H), $2.89(\mathrm{~s}, 1 \mathrm{H}), 2.44-2.16(\mathrm{~m}, 4 \mathrm{H}), 1.98-1.61(\mathrm{~m}, 11 \mathrm{H}), 1.47-1.34(\mathrm{~m}, 4 \mathrm{H}), 1.19-1.05(\mathrm{~m}, 2 \mathrm{H}), 0.89$ $(\mathrm{t}, J=6.9 \mathrm{~Hz}, 1 \mathrm{H}) ;{ }^{13} \mathrm{C} \mathrm{NMR}\left(125 \mathrm{MHz}, \mathrm{CDCl}_{3}\right) \delta: 154.29,136.69,133.17,132.68,130.21,126.85,122.06$, $121.99,121.09,111.75,111.69,111.55,110.98,110.91,98.84,98.79,98.09,98.04,77.28,76.77,68.84,68.72$, 67.61, 67.49, 64.12, 63.25, 55.59, 55.42, 42.65, 42.18, 33.21, 32.88, 31.93, 31.66, 31.16, 30.96, 29.70, 29.66, $29.37,28.60,28.51,28.35,26.58,26.48,26.39,26.25,25.92,22.70,18.94,18.03,14.12$; HR-ESIMS: $m / z$ 545.2188 [M-Cl] ${ }^{+}$(calcd. for $\mathrm{C}_{30} \mathrm{H}_{38} \mathrm{~N}_{2} \mathrm{ORh}, 545.2039$ ). Complex $2 \mathrm{f}$ is a known compound, and the NMR and high-resolution mass spectrometry of this compound were identical to those reported in the literature [36]. 2g: $90 \%$ yield; ${ }^{1} \mathrm{H}$ NMR $\left(500 \mathrm{MHz}, \mathrm{CDCl}_{3}\right) \delta: 8.04(\mathrm{~m}, 4 \mathrm{H}), 7.63(\mathrm{~m}, 4 \mathrm{H}), 7.40-7.30$ $(\mathrm{m}, 1 \mathrm{H}), 7.25-7.07(\mathrm{~m}, 3 \mathrm{H}), 6.16(\mathrm{~m}, 1 \mathrm{H}), 5.05(\mathrm{~m}, 2 \mathrm{H}), 3.75-3.55(\mathrm{~m}, 1 \mathrm{H}), 2.54-2.15(\mathrm{~m}, 3 \mathrm{H}), 1.92(\mathrm{~s}, 1 \mathrm{H})$, $1.81(\mathrm{~d}, J=7.2 \mathrm{~Hz}, 3 \mathrm{H}), 1.74-1.67(\mathrm{~m}, 1 \mathrm{H}), 1.51-1.41(\mathrm{~m}, 2 \mathrm{H}), 1.31(\mathrm{~s}, 9 \mathrm{H}), 1.21-1.05(\mathrm{~m}, 1 \mathrm{H}) ;{ }^{13} \mathrm{C}$ NMR $\left(125 \mathrm{MHz}, \mathrm{CDCl}_{3}\right) \delta: 135.28,133.64,132.76,128.65,128.46,127.81,126.98,126.86,122.47,122.39,122.35$, $122.24,113.83,113.35,110.93,110.79,99.59,98.86,97.91,77.28,76.77,70.21,67.97,67.36,67.18,66.97$, 66.86, 36.00, 35.64, 32.68, 32.06, 31.83, 31.27, 29.70, 29.49, 29.39, 29.32, 29.06, 27.72, 27.22, 15.73, 15.14, 14.12; HR-ESIMS: $m / z 539.2090$ [M-Cl] ${ }^{+}$(calcd. for $\mathrm{C}_{31} \mathrm{H}_{36} \mathrm{~N}_{2} \mathrm{Rh}$, 539.1934).

\subsection{Representative Procedure for the Rh-Catalyzed Asymmetric Arylation of Aldehyde}

The NHC-Rh complex $2 \mathrm{~g}(2.2 \mathrm{mg}, 0.00375 \mathrm{mmol})$ was weighted into $1 \mathrm{~mL}$ of DME/ $\mathrm{H}_{2} \mathrm{O}(5: 1)$ under $\mathrm{N}_{2}$. After stirring at room temperature for $5 \mathrm{~min}, \mathrm{KF}(43.6 \mathrm{mg}, 0.75 \mathrm{mmol})$, phenylboronic acid ( $30.5 \mathrm{mg}, 0.25 \mathrm{mmol})$, and 2-naphthaldehyde (19.5 mg, $0.125 \mathrm{mmol})$ were added successively. The resulting mixture was stirred at $80{ }^{\circ} \mathrm{C}$ for $12 \mathrm{~h}$. After usual work-up, purification by silica gel column (petroleum/ethyl acetate $=9 / 1$ ) afforded $4 \mathbf{a}$ as a colorless oil ( $99 \%$ yield, 35\% ee). The spectral data were comparable to those reported [38]. The ee was determined by HPLC analysis with Daicel Chiralcel OD-H (hexane $/ \mathrm{Pr}^{i} \mathrm{OH}=90 / 10$, flow rate $=0.8 \mathrm{~mL} / \mathrm{min}, t_{\mathrm{r}}$ (minor) $=19.03 \mathrm{~min}$, $t_{\mathrm{r}}($ major $\left.)=22.46 \mathrm{~min}\right)$.

Analogous compounds $4 \mathbf{b}-\mathbf{1}$ were prepared according to the similar procedure for $4 \mathbf{a}$. 4b: $97 \%$ yield, $43 \%$ ee. The spectral data were comparable to those reported [24]. The ee was determined by HPLC analysis with Daicel Chiralcel AD-H (hexane $/ \mathrm{Pr}^{i} \mathrm{OH}=90 / 10$, flow rate $=0.8 \mathrm{~mL} / \mathrm{min}$, $t_{\mathrm{r}}($ minor $)=15.0 \mathrm{~min}, t_{\mathrm{r}}($ major $\left.)=16.5 \mathrm{~min}\right) .4 \mathrm{c}: 99 \%$ yield, $37 \%$ ee. The spectral data were comparable to those reported [36]. The ee was determined by HPLC analysis with Daicel Chiralcel $\mathrm{AD}-\mathrm{H}$ (hexane $/ \mathrm{Pr}^{i} \mathrm{OH}=90 / 10$, flow rate $=0.8 \mathrm{~mL} / \mathrm{min}, t_{\mathrm{r}}($ minor $)=11.9 \mathrm{~min}, t_{\mathrm{r}}($ major $\left.)=12.8 \mathrm{~min}\right)$. $4 \mathrm{~d}$ : $85 \%$ yield, $46 \%$ ee. The spectral data were comparable to those reported [24]. The ee was determined by HPLC analysis with Daicel Chiralcel AD-H (hexane $/ \mathrm{Pr}^{i} \mathrm{OH}=90 / 10$, flow rate $=0.8 \mathrm{~mL} / \mathrm{min}$, $t_{\mathrm{r}}($ major $)=14.0 \mathrm{~min}, t_{\mathrm{r}}($ minor $\left.)=15.1 \mathrm{~min}\right) .4 \mathrm{e}: 94 \%$ yield, $40 \%$ ee. The spectral data were comparable to those reported [24]. The ee was determined by HPLC analysis with Daicel Chiralcel $\mathrm{AD}-\mathrm{H}$ (hexane $/ \mathrm{Pr}{ }^{i} \mathrm{OH}=90 / 10$, flow rate $=0.8 \mathrm{~mL} / \mathrm{min}, t_{\mathrm{r}}$ (major) $=7.5 \mathrm{~min}, t_{\mathrm{r}}$ (minor) $=8.8 \mathrm{~min}$ ). 4f: $99 \%$ yield, $28 \%$ ee. The spectral data were comparable to those reported [36]. The ee was determined by HPLC analysis with Daicel Chiralcel OD-H (hexane $/ \mathrm{Pr}^{i} \mathrm{OH}=90 / 10$, flow rate $=0.8 \mathrm{~mL} / \mathrm{min}$, $t_{\mathrm{r}}($ minor $)=10.6 \mathrm{~min}, t_{\mathrm{r}}$ (major) $\left.=12.4 \mathrm{~min}\right) .4 \mathrm{~g}: 99 \%$ yield, $36 \%$ ee. The spectral data were comparable to those reported [36]. The ee was determined by HPLC analysis with Daicel Chiralcel OD-H (hexane $/ \mathrm{Pr}^{i} \mathrm{OH}=90 / 10$, flow rate $=0.8 \mathrm{~mL} / \mathrm{min}, t_{\mathrm{r}}$ (minor) $=9.8 \mathrm{~min}, t_{\mathrm{r}}$ (major) $=10.2 \mathrm{~min}$ ). 4h: $93 \%$ yield, $38 \%$ ee. The spectral data were comparable to those reported [36]. The ee was determined 
by HPLC analysis with Daicel Chiralcel OD-H (hexane $/ \mathrm{Pr}^{i} \mathrm{OH}=90 / 10$, flow rate $=0.8 \mathrm{~mL} / \mathrm{min}$, $t_{\mathrm{r}}($ major $)=8.6 \mathrm{~min}, t_{\mathrm{r}}($ minor $\left.)=9.2 \mathrm{~min}\right) .4 \mathrm{i}: 88 \%$ yield, $28 \%$ ee. The spectral data were comparable to those reported [36]. The ee was determined by HPLC analysis with Daicel Chiralcel OD-H (hexane $/ \mathrm{Pr}^{i} \mathrm{OH}=90 / 10$, flow rate $=0.8 \mathrm{~mL} / \mathrm{min}, t_{\mathrm{r}}$ (major) $=11.2 \mathrm{~min}, t_{\mathrm{r}}($ minor $)=13.2 \mathrm{~min}$ ). $4 \mathbf{j}: 94 \%$ yield, $28 \%$ ee. The spectral data were comparable to those reported [36]. The ee was determined by HPLC analysis with Daicel Chiralcel OD-H (hexane $/ \mathrm{Pr}^{i} \mathrm{OH}=90 / 10$, flow rate $=0.8 \mathrm{~mL} / \mathrm{min}$, $t_{\mathrm{r}}($ major $)=21.5 \mathrm{~min}, t_{\mathrm{r}}($ minor $\left.)=23.8 \mathrm{~min}\right) .4 \mathbf{k}: 99 \%$ yield, $18 \%$ ee. The ee was determined by HPLC analysis with Daicel Chiralcel OD-H (hexane $/ \mathrm{Pr}^{i} \mathrm{OH}=90 / 10$, flow rate $=0.8 \mathrm{~mL} / \mathrm{min}$, $t_{\mathrm{r}}($ minor $)=11.8 \mathrm{~min}, t_{\mathrm{r}}$ (major) $\left.=12.6 \mathrm{~min}\right) . \quad 41: 98 \%$ yield, $19 \%$ ee. The ee was determined by HPLC analysis with Daicel Chiralcel OD-H (hexane $/ \mathrm{Pr}^{i} \mathrm{OH}=85 / 15$, flow rate $=0.8 \mathrm{~mL} / \mathrm{min}$, $t_{\mathrm{r}}(\operatorname{minor})=8.6 \mathrm{~min}, t_{\mathrm{r}}($ major $\left.)=9.9 \mathrm{~min}\right)$.

\section{Conclusions}

In conclusion, seven NHC-Rh complexes (2a-f) have been prepared. Their applicability in the asymmetric arylation of aromatic aldehydes has been demonstrated, and the corresponding diarylmethanols were obtained with high yields and moderate enantiomeric excesses (up to $46 \%$ ). Further work is in progress to utilize these complexes in asymmetric 1,2-addition reactions of arylboronic acids with ketones, as well as their applications in fields of nanoscience $[39,40]$.

Supplementary Materials: The following are available online at www.mdpi.com/2073-4344/6/9/132/s1, Figure S1: ${ }^{1} \mathrm{H}$ and ${ }^{13} \mathrm{C}$ NMR Spectra of compounds 2a-g, Figure S2: HR-MS Spectra for Compounds 2a-g, Figure S3: HPLC data of $\mathbf{4 a - 1 .}$

Acknowledgments: We are grateful to the National Natural Science Foundation of China (81302668) and Hangzhou Science and Technology Information Institute of China (20150633B45).

Author Contributions: Jie Li and Jianyou Shi conceived and designed the experiments; Weiping He and Bihui Zhou performed the experiments and analyzed the data; Jie Li and Jianyou Shi wrote the paper.

Conflicts of Interest: The authors declare no conflict of interest.

\section{References}

1. Herrmann, W.A.; Köcher, C. N-heterocyclic carbenes. Angew. Chem. Int. Ed. 1997, 36, 2162-2187. [CrossRef]

2. Bourissou, D.; Guerret, O.; Gabbaï, F.P.; Bertrand, G. Stable carbenes. Chem. Rev. 2000, 100, 39-92. [CrossRef] [PubMed]

3. Cavell, K.J.; McGuinness, D.S. Redox processes involving hydrocarbylmetal (N-heterocyclic carbene) complexes and associated imidazolium salts: Ramifications for catalysis. Coord. Chem. Rev. 2004, 248, 671-681. [CrossRef]

4. Peris, E.; Crabtree, R.H. Recent homogeneous catalytic applications of chelate and pincer $N$-heterocyclic carbenes. Coord. Chem. Rev. 2004, 248, 2239-2246. [CrossRef]

5. Crudden, C.M.; Allen, D.P. Stability and reactivity of N-heterocyclic carbene complexes. Coord. Chem. Rev. 2004, 248, 2247-2273. [CrossRef]

6. Pugh, D.; Danopoulos, A.A. Metal complexes with 'pincer'-type ligands incorporating N-heterocyclic carbene functionalities. Coord. Chem. Rev. 2007, 251, 610-641. [CrossRef]

7. Mata, J.A.; Poyatos, M.; Peris, E. Structural and catalytic properties of chelating bis- and tris- $N$-heterocyclic carbenes. Coord.Chem. Rev. 2007, 251, 841-859. [CrossRef]

8. Sommer, W.J.; Weck, M. Supported N-heterocyclic carbene complexes in catalysis. Coord. Chem. Rev. 2007, 251, 860-873. [CrossRef]

9. Hahn, F.E.; Jahnke, M.C. Heterocyclic carbenes: Synthesis and coordination chemistry. Angew. Chem. Int. Ed. 2008, 47, 3122-3172. [CrossRef] [PubMed]

10. Hopkinson, M.N.; Richter, C.; Schedler, M.; Glorius, F. An overview of N-heterocyclic carbenes. Nature 2014, 510, 485-496. [CrossRef] [PubMed]

11. Perry, M.C.; Burgess, K. Chiral N-heterocyclic carbene-transition metal complexes in asymmetric catalysis. Tetrahedron 2003, 14, 951-961. [CrossRef] 
12. César, V.; Bellemin-Laponnaz, S.; Gade, L.H. Chiral N-heterocyclic carbenes as stereodirecting ligands in asymmetric catalysis. Chem. Soc. Rev. 2004, 33, 619-636. [CrossRef] [PubMed]

13. Douthwaite, R.E. Metal-mediated asymmetric alkylation using chiral $N$-heterocyclic carbenes derived from chiral amines. Coord. Chem. Rev. 2007, 251, 702-717. [CrossRef]

14. Gade, L.H.; Bellemin-Laponnaz, S. Mixed oxazoline-carbenes as stereodirecting ligands for asymmetric catalysis. Coord. Chem. Rev. 2007, 251, 718-725. [CrossRef]

15. Snead, D.R.; Seo, H.; Hong, S. Recent developments of chiral diaminocarbene-metal complexes for asymmetric catalysis. Curr. Org. Chem. 2008, 12, 1370-1387. [CrossRef]

16. Wang, F.J.; Liu, L.J.; Wang, W.F.; Li, S.K.; Shi, M. Chiral NHC-metal-based asymmetric catalysis. Coord. Chem. Rev. 2012, 256, 804-853. [CrossRef]

17. Meguro, K.; Aizawa, M.; Sohda, T.; Kawamatsu, Y.; Nagaoka, A. New 1,4-dihydropyridine derivatives with potent and long-lasting hypotensive effect. Chem. Pharm. Bull. 1985, 33, 3787-3797. [CrossRef]

18. Stanchev, S.; Rakovska, R.; Berova, N.; Snatzke, G. Synthesis, absolute configuration and circular dichroism of some diarylmethane derivatives. Tetrahedron 1995, 6, 183-198. [CrossRef]

19. Bolshan, Y.; Chen, C.; Chilenski, J.R.; Gosselin, F.; Mathre, D.J.; O’Shea, P.D.; Roy, A.; Tillyer, R.D. Nucleophilic displacement at benzhydryl centers: Asymmetric synthesis of 1,1-diarylalkyl derivatives. Org. Lett. 2004, 6, 111-114. [CrossRef] [PubMed]

20. Radin, N.S. Sphingolipids as coenzymes in anion transfer and tumor death. Bioorganic Medicinal Chem. 2004, 12, 6029-6037. [CrossRef]

21. Fang, L.; Changduo, P.; Jiang, C. Progress in the addition of arylboronic acids to aromatic aldehydes and ketone. Chin. J. Org. Chem. 2010, 30, 633-639.

22. Sakai, M.; Ueda, M.; Miyaura, N. Rhodium-catalyzed addition of organoboronic acids to aldehydes. Angew. Chem. Int. Ed. 1998, 37, 3279-3281. [CrossRef]

23. Shintani, R.; Inoue, M.; Hayashi, T. Rhodium-catalyzed asymmetric addition of aryl- and alkenylboronic acids to isatins. Angew. Chem. Int. Ed. 2006, 45, 3353-3356. [CrossRef] [PubMed]

24. Duan, H.F.; Xie, J.H.; Shi, W.J.; Zhang, Q.; Zhou, Q.L. Enantioselective rhodium-catalyzed addition of arylboronic acids to aldehydes using chiral spiro monophosphite ligands. Org. Lett. 2006, 8, 1479-1481. [CrossRef] [PubMed]

25. Duan, H.F.; Xie, J.H.; Qiao, X.C.; Wang, L.X.; Zhou, Q.L. Enantioselective rhodium-catalyzed addition ofarylboronic acids to $\alpha$-ketoesters. Angew. Chem. Int. Ed. 2008, 47, 4351-4353. [CrossRef] [PubMed]

26. Yamamoto, Y.; Kurihara, K.; Miyaura, N. Me-bipam for enantioselective ruthenium(II)-catalyzed arylation of aldehydes with arylboronic acids. Angew. Chem. Int. Ed. 2009, 121, 4478-4480. [CrossRef]

27. Morikawa, S.; Michigami, K.; Amii, H. Novel axially chiral phosphine ligand with a fluoro alcohol moiety for Rh-catalyzed asymmetric arylation of aromatic aldehydes. Org. Lett. 2010, 12, 2520-2523. [CrossRef] [PubMed]

28. Zhang, R.; Xu, Q.; Zhang, X.C.; Zhang, T.; Shi, M. Axially chiral $\mathrm{C}_{2}$-symmetric N-heterocyclic carbene (NHC) palladium complexes-catalyzed asymmetric arylation of aldehydes with arylboronic acids. Tetrahedron 2010, 21, 1928-1935. [CrossRef]

29. Shintani, R.; Takatsu, K.; Hayashi, T. Copper-catalyzed asymmetric addition of arylboronates to isatins: a catalytic cycle involving alkox Copper intermediates. Chem. Commun. 2010, 46, 6822-6824. [CrossRef] [PubMed]

30. Liu, Z.; Gu, P.; Shi, M.; McDowell, P.; Li, G.G. Catalytic asymmetric addition of arylboronic acids to isatins using $\mathrm{C}_{2}$-symmetric cationic $\mathrm{N}$-heterocyclic carbenes (NHCs) $\mathrm{Pd}^{2+}$ diaqua complexes as catalysts. Org. Lett. 2011, 13, 2314-2317. [CrossRef] [PubMed]

31. Arao, T.; Sato, K.; Kondo, K.; Aoyama, T. Function of an N-heterocyclic carbene ligand based on concept of chiral mimetic. Chem. Pharm. Bull. 2006, 54, 1576-1581. [CrossRef] [PubMed]

32. Ma, Q.S.; Ma, Y.D.; Liu, X.; Duan, W.Z.; Qu, B.; Song, C. Planar chiral imidazolium salts based on [2.2]paracyclophane in the asymmetric rhodium-catalyzed 1,2-addition of arylboronic acids to aldehydes. Tetrahedron 2010, 21, 292-298. [CrossRef]

33. Duan, W.Z.; Ma, Y.D.; Qu, B.; Zhao, L.; Chen, J.Q.; Song, C. Synthesis of new alkoxy/sulfonate-substituted carbene precursors derived from [2.2]paracyclophane and their application in the asymmetric arylation of aldehydes. Tetrahedron 2012, 23, 1369-1375. [CrossRef] 
34. Duan, W.Z.; Ma, Y.D.; He, F.Y.; Zhao, L.; Chen, J.Q.; Song, C. Synthesis of novel planar chiral Ag and Rh $N$-heterocyclic carbine complexes derived from [2.2]paracyclophane and their application in ultrasound assisted asymmetric addition reactions of organoboronic acids to aldehydes. Tetrahedron 2013, 24, 241-248. [CrossRef]

35. Chen, J.Q.; Yang, S.B.; Chen, Z.; Song, C.; Ma, Y.D. Synthesis of novel macrocyclic planar chiral carbene-Ag complexes derived from [2.2]paracyclophane for Rh-catalyzed asymmetric 1,2-additions of arylboronic acids to aromatic aldehydes. Tetrahedron 2015, 26, 288-295. [CrossRef]

36. He, W.P.; Zhou, B.H.; Zhou, Y.L.; Li, X.R.; Fan, L.M.; Shou, H.W.; Li, J. Synthesis of new benzimidazolium salts and their application in the asymmetric arylation of aldehydes. Tetrahedron Lett. 2016, 57, 3152-3155. [CrossRef]

37. He, W.P.; Zhao, W.; Zhou, B.H.; Liu, H.F.; Li, X.R.; Li, L.L.; Li, J.; Shi, J.Y. Synthesis of $C_{2}$-symmetric benzimidazolium salts and their application in palladium-catalyzed enantioselective intramolecular $\alpha$-arylation of amides. Molecules 2016, 21, 742. [CrossRef] [PubMed]

38. Yang, Y.X.; Liu, Y.; Zhang, L.; Jia, Y.E.; Wang, P.; Zhuo, F.F.; An, X.T.; Da, C.S. Aryl bromides as inexpensive starting materials in the catalytic enantioselective arylation of aryl aldehydes: The additive TMEDA enhances the enantioselectivity. J. Org. Chem. 2014, 79, 10696-10702. [CrossRef] [PubMed]

39. Caricato, M.; Sharma, A.K.; Coluccini, C.; Pasini, D. Nanostructuring with chirality: Binaphthyl-based synthons for the production of functional oriented nanomaterials. Nanoscale 2014, 6, 7165-7174. [CrossRef]

40. Caricato, M.; Delforge, A.; Bonifazi, D.; Dondi, D.; Mazzanti, A.; Pasini, D. Chiral nanostructuring of multivalent macrocycles in solution and on surfaces. Org. Biomol. Chem. 2015, 13, 3593-3601. [CrossRef] [PubMed]

(C) 2016 by the authors; licensee MDPI, Basel, Switzerland. This article is an open access article distributed under the terms and conditions of the Creative Commons Attribution (CC-BY) license (http://creativecommons.org/licenses/by/4.0/). 